\title{
Surface Modification of Powder Metallurgy Titanium by Colloidal Techniques and Diffusion Processes for Biomedical...
}

Article in Advanced Engineering Materials · July 2016

DOI: 10.1002 /adem.201600207

CITATIONS

0

7 authors, including:

\section{Begona Ferrari}

Institute of Ceramics and Glass

100 PUBLICATIONS 1,029 CITATIONS

SEE PROFILE

\section{Sophia A. Tsipas}

University Carlos III de Madrid

41 PUBLICATIONS 400 CITATIONS

SEE PROFILE
READS

23

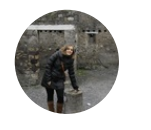

Y. Castro

Spanish National Research Council

59 PUBLICATIONS 837 CITATIONS

SEE PROFILE

E. Gordo

University Carlos III de Madrid

143 PUBLICATIONS 797 CITATIONS

SEE PROFILE 


\title{
Surface Modification of Powder Metallurgy Titanium by Colloidal Techniques and Diffusion Processes for Biomedical Applications**
}

\author{
By Julia Ureña, ${ }^{*}$ Carlos Mendoza, Begoña Ferrari, Yolanda Castro, Sophia A. Tsipas, \\ Antonia Jiménez-Morales and Elena Gordo
}

This contribution focuses on modifying the composition and surface characteristics of powder metallurgy titanium substrates to improve their wear resistance and to reduce the surface Young's modulus, while maintaining the corrosion resistance and biocompatibility. The modification consists of Mo and TiN coatings produced by colloidal techniques which are deposited by electrophoretic deposition (EPD) or spraying onto green or sintered titanium substrates. The final surface structures are formed during a heat treatment, during which diffusion and consolidation of the layers take place. The surfaces are characterised by XRD, SEM, and micro-indentation; showing diffusion areas of between 40 and 85 microns with clear microstructural changes as well as higher hardness values.

\section{Introduction}

Titanium and its alloys are materials known for their attractive combination of properties. However, although they are considered as the preferred metals for biomedical applications, the long-term performance of surgical implants is often limited by their surface properties like low wear and corrosion resistance which results in the release of incompatible metal ions into the human body. $\underline{[1,2]}^{[1}$ Other aspects related to the poor tribological properties of $\mathrm{Ti}$ alloys, such as high friction coefficient and wear debris formation, can produce inflammatory reactions and may contribute to loosening of the implant and implant failure. ${ }^{[3]}$ Surface modification is the best alternative for improving corrosion and wear resistance, osseointegration rate, biocompatibility, and aesthetic aspects. ${ }^{[4,5]}$ One of the measures which helps improve

[*] Dr. J. Ureña, Dr. S. A. Tsipas, Prof. A. Jiménez-Morales, Prof. E. Gordo

Carlos III University, IAAB, Avda. Universidad 30, 28911 Leganés, Spain

E-mail: jurena@pa.uc3m.es

Dr. C. Mendoza, Dr. B. Ferrari, Dr. Y. Castro

Institute of Ceramic and Glass (CSIC), Campus de Cantoblanco, c/ Kelsen 5, 28049 Madrid, Spain

[**] Funds provided by Spanish Government (program MINECO, ref. MAT2012-38650-C02-01) and Regional Government of Madrid (program MULTIMAT-CHALLENGE, ref. S2013/ MIT-2862). the wear behavior of the Ti alloys is surface hardening by means of several techniques such as ion implantation, plasma spray, chemical surface treatments like nitriding, carburization, and boriding. ${ }^{[6,7]}$

Titanium nitride (TiN) coatings have been studied for many years because of their good wear and corrosion resistance. ${ }^{[4,5]}$ Apart from that, its most important application is as a hard coating for cutting tools, although it is also employed for surgical tools and implants. $\underline{[8]}$ TiN coatings are usually produced by nitridation, physical vapour deposition (PVD) and chemical vapour deposition (CVD); which are high-cost techniques due to the extreme conditions of pressure, energy, and/or temperature that are required. ${ }^{[9]}$ In this sense, there is a great interest in the synthesis and deposition of $\mathrm{TiN}$ nanoparticles at low temperature, using techniques such as sol-gel and electrodeposition. ${ }^{[10]}$ There is an increasing interest in electrophoretic deposition (EPD) among the techniques considered because of its low cost, simplicity, versatility, and capability of producing dense, fine, and deep ceramic coatings reproducing the substrate shape in addition to being environmentally friendly. ${ }^{[10,11]}$

Another main requirement for a biomaterial is a low Young's modulus in order to reduce the stress shielding effect. Stress shielding occurs when there is biomechanical incompatibility of the biomaterial due to its higher stiffness with respect to the bone which leads to death of bone cells. ${ }^{[1]}$ The Young's modulus of Ti alloys depends on the phases present in their microstructure and a reduction of the Young's modulus can be achieved by using Beta $(\beta)$ titanium alloys, 
which is why they are widely studied..$^{[7,12]}$ These kind of alloys exhibit lower Young's modulus than other typical biomedical alloys like Co-Cr-Mo alloys (210 GPa), stainless steel $(200 \mathrm{GPa})$, or Ti-6Al-4V (110 GPa). ${ }^{[13]}$

Molybdenum is among the non-toxic and non-allergenic $\beta$-stabilizer elements whose addition improves the mechanical performance of the Ti alloys increasing their corrosion resistance and obtaining values of the elastic modulus as low as $50 \mathrm{GPa}$, much closer to that of bone (10-40 GPa). ${ }^{[14-16]}$ The relevance of the powder metallurgy production in Tibased alloys is shown in recent works with niobium and molybdenum as $\beta$-stabilizer elements for the development of biomaterials with low Young's modulus. ${ }^{[17,18]}$

The aim of this work is to study the surface modification of powder metallurgy titanium substrates by means of different techniques and coatings to improve hardness and wear resistance and to reduce the Young's modulus. On one hand, diffusion processes are used to modify the composition and microstructural features of titanium substrates by depositing a layer of Mo particles on green or sintered substrates; in the first case, the co-sintering of the deposited layer and green substrate is studied, whereas in the second case, a heat treatment is applied to promote the diffusion of the Mo into the sintered substrate. On the other hand, a layer of TiN nanoparticles is deposited by EPD onto the sintered Ti substrate both after and before Mo deposition. All the materials produced are characterized and compared in terms of microstructural features and hardness. The results aim to shed light on the design and the surface modification of Ti-based materials through powder metallurgy with interesting biomedical properties for further investigations.

\section{Experimental Section}

\subsection{Preparation of Titanium Substrates}

Substrates were produced using hydride-dehydride (HDH) commercially pure titanium powder (CPTi grade 4) (GfE Metalle und Materialien GmbH, Germany) with particle size below $75 \mu \mathrm{m}$. Green and sintered substrates were fabricated following a route established in previous works. $[19,20]$ Green substrates were prepared by uniaxial pressing at $600 \mathrm{MPa}$ employing a cylindrical mold of $16 \mathrm{~mm}$ in diameter with lubricated walls using zinc stearate. For the sintered substrates, after the pressing step, a sintering cycle was performed in high vacuum $\left(10^{-5} \mathrm{mbar}\right)$ at $1250^{\circ} \mathrm{C}$ for $2 \mathrm{~h}$ with a heating and cooling rate of $5^{\circ} \mathrm{C} \mathrm{min}^{-1}$. Pieces were placed inside the furnace in a bed of zirconia balls as a support. Table 1 shows dimensions and densities of the green and sintered titanium substrates.

The surfaces of the green titanium samples did not need any surface preparation before the molybdenum deposition. However, the sintered titanium substrates were prepared following a metallographic route for the subsequent coating. This preparation involved grinding using sandpaper from 180 to 1000 grade, washing in distilled water, cleaning in
Table 1. Substrate materials characteristics.

\begin{tabular}{lccc} 
Material & Substrate & Density $\left[\mathrm{g} \mathrm{cm}^{-3}\right]$ & Relative density [\%] \\
\hline CPTi & Green & $3.8 \pm 0.1$ & 84 \\
CPTi & Sintered & $4.3 \pm 0.1$ & 95
\end{tabular}

ultrasonic bath with ethanol, and drying using hot air. For the modification with TiN, an additional step of polishing with 9, 1, and 0.3 microns alumina suspensions was necessary.

\subsection{Titanium Surface Modification. Coatings, Techniques, and Diffusion Process}

Two techniques were used to modify the Ti substrates, creating four different types of samples. The schematic in Figure 1 summarizes the routes followed; the samples designed and the nomenclature followed from now on for each sample type is specified.

\subsubsection{Titanium Nitride (TiN) Layer by Electrophoretic Deposition (EPD)}

TiN layers were deposited by EPD onto polished sintered titanium substrates. TiN nanoparticles had a mean particle size of $20 \mathrm{~nm}$ and were supplied by Hefei Kaier Nanometer Energy \& Technology, China. For the coating process, a TiN suspension $\left(0.1 \mathrm{~g} \mathrm{~L}^{-1}\right)$ was prepared using isopropyl alcohol (99.7\%, Panreac, Spain) as a solvent and polyethylenimine (1.5 $\mathrm{wt} \%$ ) (PEI, Sigma-Aldrich, Germany) as a stabilizer. ${ }^{[10]}$ A schematic of the EPD and the substrate at different stages of the whole process is shown in Figure 2.

After TiN deposition, which took place for $5 \mathrm{~min}$, green coatings were left to dry at room conditions. For the layer consolidation, a thermal treatment was performed in vacuum atmosphere at $1200^{\circ} \mathrm{C}$ for $1 \mathrm{~h}$ with heating and cooling rates of $5^{\circ} \mathrm{C} \mathrm{min}^{-1}$.

\subsubsection{Molybdenum (Mo) Coating by Spraying}

An aqueous suspension of molybdenum powder with a particle size between 1 and $2 \mu \mathrm{m}$ (Sigma-Aldrich, Germany) was prepared. A suspension containing $10 \%$ volume Mo particles was adjusted to $\mathrm{pH} 10$ by tetramethylammonium hydroxide (HTMA). After a dilution $\left(0.1 \mathrm{~g} \mathrm{~L}^{-1}\right)$ in $\mathrm{KCl}$ $10^{-2} \mathrm{~N}$, the suspension stability was evaluated as a function of its zeta potential at the $\mathrm{pH}$ of 2, 4, 6, 8, 10, and 12 (adjusted by $\mathrm{HTMA}$ and $\mathrm{HNO}_{3}$ ) (Figure 3). Before each zeta potential measure, the different $\mathrm{pH}$ suspensions were submitted to an ultrasonic probe for $30 \mathrm{~s}$ at $55 \%$ power in order to homogenize and break unwanted agglomerates. The measure of the zeta potential was performed with the microelectrophoresis technique by Zeta-Meter 3.0 equipment. Two characteristic points define the surface state for a particle in a liquid medium: isoelectric point (value of $\mathrm{pH}$ which 


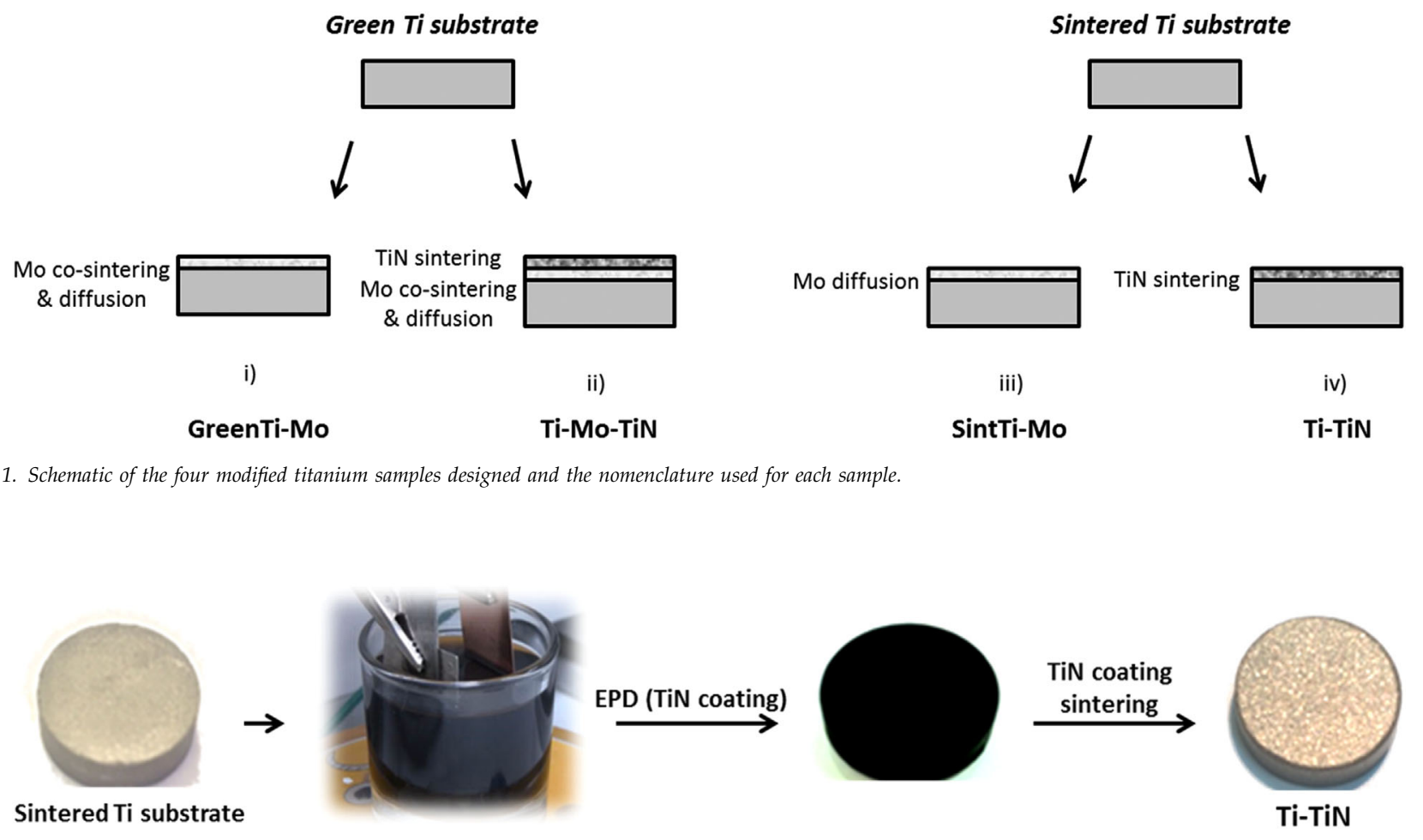

Fig. 2. EPD process for Ti-TiN sample.

the zeta potential is zero) and point of charge zero (where the positive and negative surface charges are the same). A suspension can be considered stable if its zeta potential presents an absolute value superior to $20 \mathrm{mV}^{[21]}$ In Figure 3, it can be observed that, with the exception of the most acid $\mathrm{pH}$, the zeta potential exhibits an absolute value higher than $20 \mathrm{mV}$ for all the $\mathrm{pH}$ values tested; therefore, determining the $\mathrm{pH}$ range for suspension stability. After the preliminary

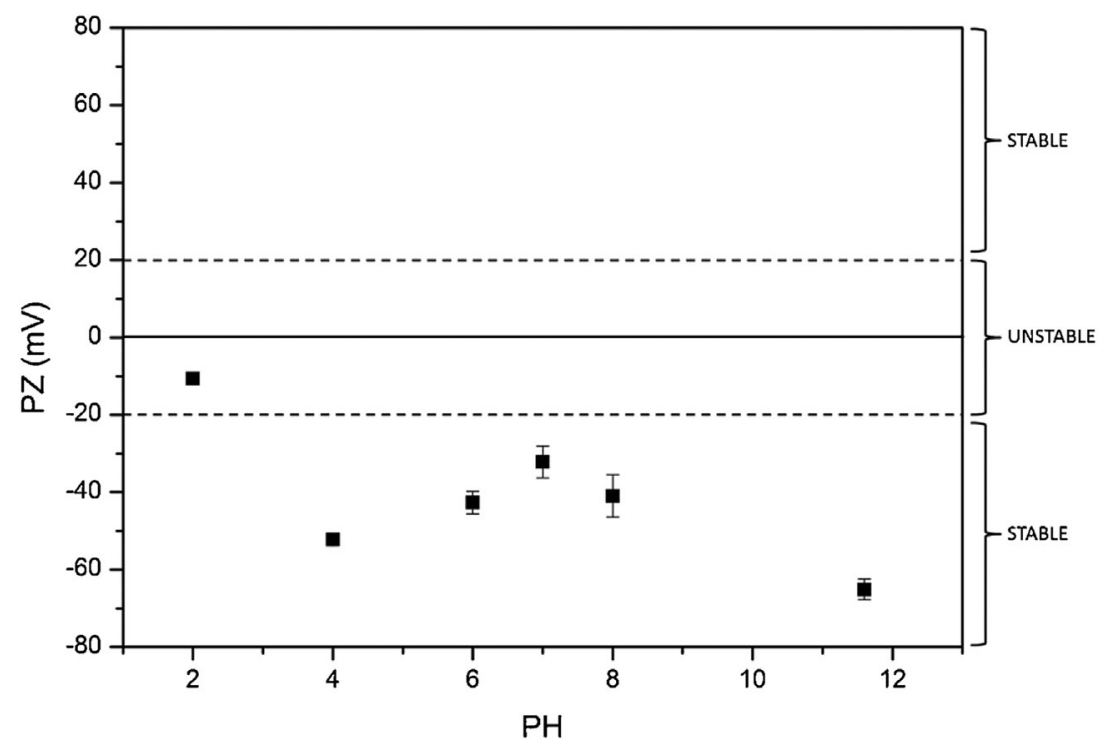

Fig. 3. PZ variation depending on the $p H$ study for the preparation of the aqueous suspension was finalized, $2 \mathrm{~h}$ in a ball mill for homogenization was necessary before spraying the suspension onto the green and sintered titanium substrates.

After deposition of the Mo particles, a thermal treatment was applied for the co-sintering and diffusion of Mo coatings in green and sintered substrates, respectively. The cycle was performed under vacuum $\left(10^{-5} \mathrm{mbar}\right)$ and consisted on heating to $1100^{\circ} \mathrm{C}$, a dwell time of $3 \mathrm{~h}$, and cooling back at $5^{\circ} \mathrm{C} \mathrm{min}^{-1}$.

\subsubsection{Molybdenum-Titanium Nitride (Mo-TiN) Multilayer by Combination of Spraying and Electrophoretic Deposition (EPD)}

The combination of these two coatings was applied onto the green titanium substrate, creating a Mo-TiN diffusion multilayer. First, the molybdenum suspension was sprayed onto the green substrate, as it was detailed before. After the heat treatment to achieve the first surface modification by Mo layer deposition, a soft grinding step on sandpaper of 1000 grade was carried out to remove the remains of undiffused molybdenum; leaving the surface clean and ready for the TiN deposition by EPD. The final structure was obtained after the heat treatment corresponding to the TiN layer sintering. 


\subsection{Surface and Microstructural Characterization}

The modified surfaces were characterized by scanning electron microscopy (SEM), $X$-ray diffraction (XRD), and Micro Vickers hardness tests.

Modified samples by TiN, Mo, and MoTiN and a titanium sample as a control sample, were submitted to surface and microstructural characterization by SEM. Images were obtained through a microscope Philips XL-30 with filament of thermionic emission of tungsten using a power of $10 \mathrm{KV}$ accelerators. The computer equipped with EDAX DX-4 analyzer spectroscopy scattered X-ray energy (EDS), allowed the semi-quantitative analysis of the chemical composition.

Surfaces were analyzed by X-ray diffraction by means of a Philips X'Pert diffractometer and the data were acquired and processed through software HighScore. A fixed wavelength with a monochromatic X-ray beam $(\mathrm{Cu} \mathrm{K} \alpha$ radiation, $\lambda=1.5405 \AA)$ was used and angles of diffraction $(\theta)$ in accordance with the Bragg law. Measurements were performed under conditions of voltage $40 \mathrm{kV}$ and current $40 \mathrm{~mA}$, with a step of $0.02^{\circ}$ and a time of $2.4 \mathrm{~s}$. Diffraction peaks obtained were identified and compared with those of patterns PCPDFWIN database.

Hardness measurements were performed on the different samples designed. Firstly, regarding the TiN coating, nanoindentation tests were performed onto TiN surfaces as well as on the control titanium sample for comparison. The CETR Bruker nanoindenter was used with Berkovich diamond tip of radius of $100 \mathrm{~nm}$, increasing the load from 0 to $500 \mathrm{mN}$. Secondly, micro Vickers hardness tests were carried out in three different parts: surface, diffusion layer, and substrate. A Zwick Roell micro-hardness tester with a diamond tip and load of $100 \mathrm{~g}(0.1 \mathrm{HV})$ was used and the data were analyzed by the hardness testing software $\mathrm{ZH} \mu \mathrm{HD}$.

\section{Results and Discussion}

\subsection{Characterization of External Surfaces}

The SEM micrographs of the different samples' surfaces are shown in Figure 4. Figure 4a shows the surface corresponding to the polished sintered titanium sample (Ti), as prepared without modification, in order to observe the surface aspect before coating and for comparison purposes. TiN coating deposited by EPD, corresponding to the Ti-TiN sample, is presented in Figure $4 \mathrm{~b}$. In a previous work, a similar TiN coating morphology was obtained by electrophoretic deposition on a stainless steel substrate using the same conditions. ${ }^{[22]}$ The typical morphology of a sprayed coating is shown in Figure $4 c$; this is the case of a molybdenum coating on a green
Ti substrate (GreenTi-Mo) after the diffusion treatment. It can be seen that the top surface is constituted of uneven particles and pores which provide some degree of roughness. Other authors show some similar surface morphologies of coatings at different spray conditions with which our surface can be compared. ${ }^{[23]}$ Finally, Figure $4 \mathrm{~d}$ shows the surface of the multilayer Mo-TiN coating corresponding to the Ti-Mo-TiN sample.

Figure 5 shows the X-ray diffraction for the titanium nitride-coated sample (Figure 5a); and the Mo-TiN multilayer and molybdenized samples (Figure 5b). The XRD pattern of the TiN coating exhibits three peaks corresponding to TiN (JCPDS No. 00-038-1420). The first intense peak appears at $2 \theta-36^{\circ}$, the second important at $2 \theta-42^{\circ}$, and one less intense at $2 \theta-62^{\circ}$. These peaks can also be observed in the Mo-TiN multilayer material in Figure $5 b$, as well as peaks corresponding to $\mathrm{Mo}_{2} \mathrm{~N}$ compound $\left(2 \theta-37^{\circ}, 2 \theta-62^{\circ}, 2 \theta-76^{\circ}\right)$ and $\mathrm{Ti}_{2} \mathrm{~N}\left(2 \theta-44^{\circ}, 2 \theta-78^{\circ}\right)$. The molybdenized surface (Figure $5 \mathrm{~b}$ ) presents the peaks related to $\alpha$ titanium and molybdenum (also present in Ti-Mo-TiN material), which are in order of appearance, $2 \theta-38^{\circ}, 2 \theta-52^{\circ}, 2 \theta-69^{\circ}$, and $2 \theta-40^{\circ}, 2 \theta-58^{\circ}$, $2 \theta-74^{\circ}$; respectively. The $\beta$ titanium peak $2 \theta-39^{\circ}$ also is present in the three molybdenized samples, which indicates the partial transformation process of $\alpha$ titanium into $\beta$ titanium that occurred during the thermal treatment applied for the Mo diffusion. No difference is appreciated between the spectra corresponding to the SintTi-Mo sample and the spectra corresponding to the GreenTi-Mo sample, both of them exhibit the peak for the $\beta$ titanium. 
a)

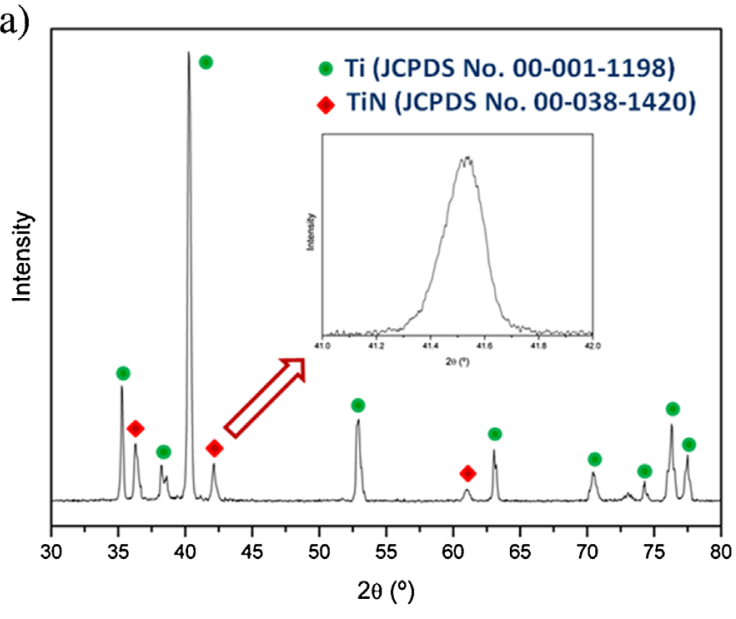

b)

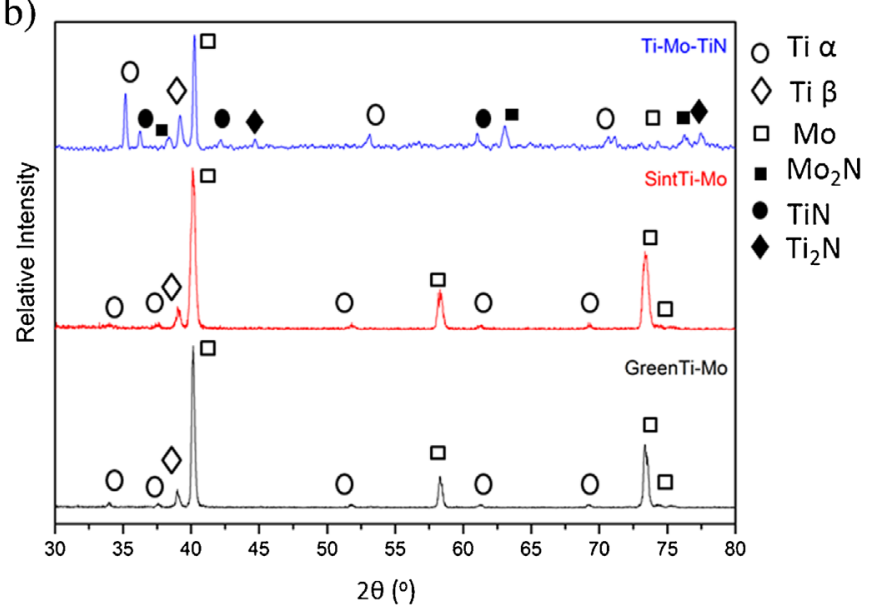

Fig. 5. X-ray diffraction patterns of the coated surfaces. (a) Titanium nitride surface on sample Ti-TiN. (b) Mo-N layer on sample Ti-Mo-TiN; and the molybdenized surface on samples SintTi-Mo and GreenTi-Mo.

Hardness of the new designed materials was measured by nano-indentation tests on polished titanium surface (Figure 4a) and TiN coating (Figure 4b); and by micro-Vickers hardness on polished titanium surface (Figure 4a), molybdenized surface (Figure 4c), and the multilayer Mo-TiN (Figure 4d); results are shown in Table 2.

\subsection{Characterization of the Cross-Section Surfaces}

Microstructural changes for the four modified materials designed are shown in Figure 6, at lower (left) and higher (right) magnification. As it can be seen in Figure $6 a-c$, a gradient of composition was obtained due to the molybdenum diffusion during the heat treatment, creating diffusion areas of different sizes between 45 and $115 \mu \mathrm{m}$. Mo introduction leads the microstructural change of the $\alpha$ single-phase titanium into two-phase region formed by colonies of $\alpha+\beta$. Depending on the initial state of the substrate, differences are observed in the diffusion areas. In the case of the surface modification performed on green substrate (GreenTi-Mo), the new created layer shows a diffusion layer depth of $85 \mu \mathrm{m}$ from the surface (Figure 6a); which suggests that achieving the surface modification in only one step during a co-sintering process is possible. Figure $6 \mathrm{~b}$ presents the surface modification carried out on

Table 2. Micro Vickers hardness (HV 0.1) of cp Ti, Ti-Mo, and Ti-Mo-TiN samples.

\begin{tabular}{lccc} 
& & \multicolumn{2}{c}{ Transversal section } \\
\cline { 3 - 4 } & & & \\
\cline { 3 - 4 } Samples & Top surface & $\alpha-\beta$ area & $\alpha$ area \\
& {$[0.1 \mathrm{HV}]$} & {$[0.1 \mathrm{HV}]$} & {$[0.1 \mathrm{HV}]$} \\
\hline CP Titanium & $279 \pm 10$ & - & $271 \pm 15$ \\
GreenTi-Mo & $370 \pm 17$ & $281 \pm 28$ & $273 \pm 26$ \\
SintTi-Mo & $430 \pm 21$ & $429 \pm 39$ & $279 \pm 20$ \\
Ti-Mo-TiN & - & $420 \pm 13$ & $293 \pm 14$ \\
& & &
\end{tabular}

the sintered substrate, SintTi-Mo, which presents a uniform and compact diffusion area with depth of $45 \mu \mathrm{m}$ along the sample. Figure $6 c$, the modified material by introduction of molybdenum plus a nitride titanium coating, Ti-Mo-TiN, shows the deepest diffusion area of $115 \mu \mathrm{m}$. Brightest zones in Figure $6 a$ and $b$ indicate rests of undiffused molybdenum and it is possible to see major porosity in the GreenTi-Mo sample. Microstructures and diffusion layer depths are very reproducible. In previous published works with similar conditions of diffusion temperature, the diffusion layer obtained presents a thickness around $200 \mu \mathrm{m}$. However, the diffusion time employed is longer; requiring higher level of energy and the use of an activator. $\frac{[15]}{2}$ On the other hand, it has been demonstrated that increasing the energy supply to introduce an alloying element does not mean an increase in the content of that alloying element. ${ }^{[24]}$ Figure $6 \mathrm{~d}$ shows the consolidated 5 micron TiN layer obtained by electrophoretic deposition onto the polished titanium substrate, Ti-TiN. As it can be seen, it is a well-defined coating with a continuous and homogeneous character. The aspect and thickness for this TiN coating can be compared to that presented in Mendoza et al. ${ }^{[22]}$ Both coatings, prepared by electrophoretic deposition, provide similar TiN layers in a range of 5-20 $\mu \mathrm{m}$ of thickness, depending on the substrate material.

The microstructural changes depend on the distance from the surface, or the depth inside the sample, and there is a correlation between the Mo content and hardness. In Figure 6, it is clear that closer to the external surface, the $\alpha+\beta$ layers are thinner and spaced closer together, becoming apparently a single phase rich in Mo.

Figure 7 shows the different $(\alpha-\beta)$ diffusion area depths and Mo content variation as a function of distance from surface obtained from EDS analysis on transversal sections of the samples (Figure 6). It can be observed that in the $(\alpha-\beta)$ diffusion area of GreenTi-Mo that has a thickness of $85 \mu \mathrm{m}$ Mo 
a)

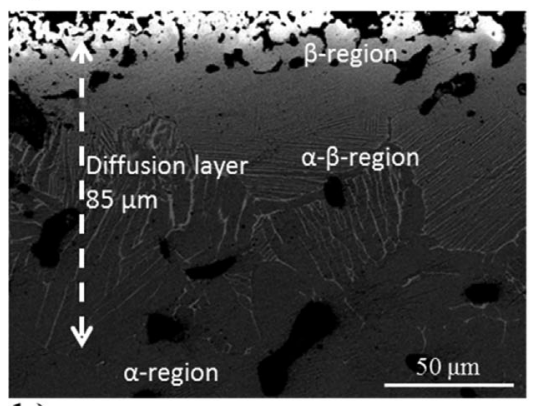

b)

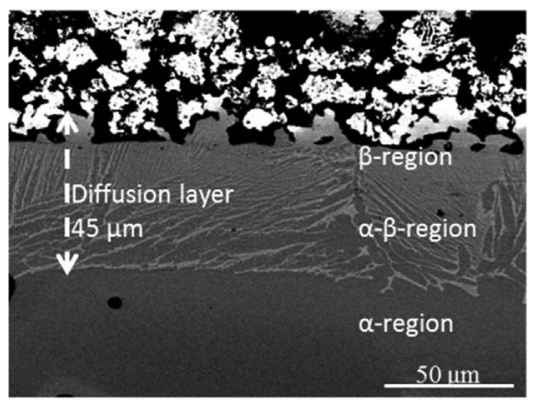

c)

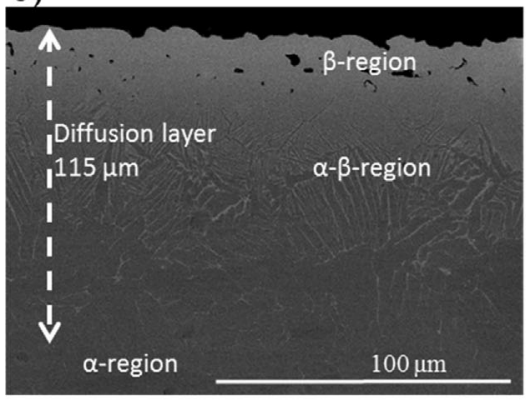

d)
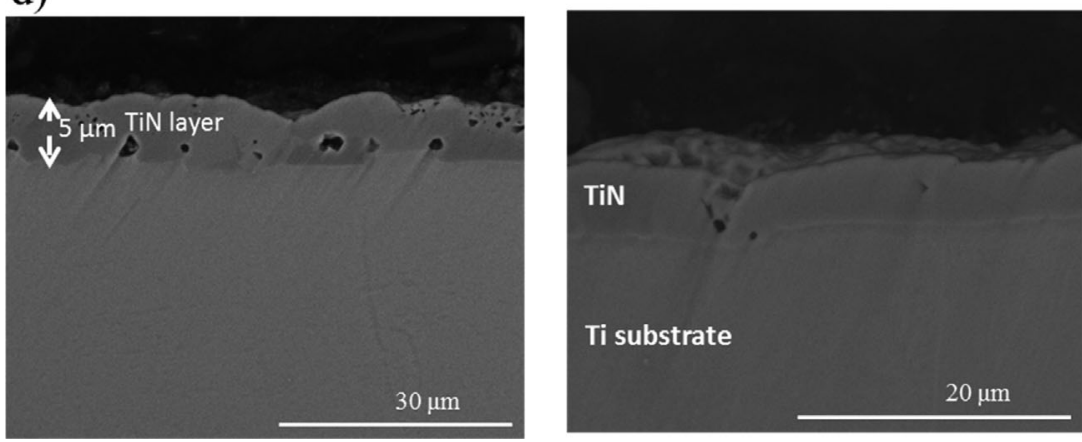

Fig. 6. Cross-sectional SEM micrographs of the diffusion areas obtained for: (a) GreenTi-Mo. (b) SintTi-Mo. (c) Ti-Mo-TiN. (d) Ti-TiN.

is present in 9 and $2 \mathrm{wt} \%$, at a distance of 40 and $80 \mu \mathrm{m}$, respectively. SintTi-Mo sample shows a narrower $(\alpha-\beta)$ diffusion area of $45 \mu \mathrm{m}$ in which Mo is in 7 and $4 \mathrm{wt} \%$ for 20 and $40 \mu \mathrm{m}$ distance from surface, respectively. The multilayer sample, Ti-Mo-TiN, exhibits the thickest $(\alpha-\beta)$ diffusion area of $115 \mu \mathrm{m}$ where the Mo contents are 17, 9, and $2 \mathrm{wt} \%$ for the distances of 10,50 , and $100 \mu \mathrm{m}$, respectively. These different quantities of Mo introduced are similar to those reported in the study mentioned before, from 2 to $18 \mathrm{Mo} \mathrm{wt} \%$. ${ }^{[13]}$

Nano-indentation measures were carried out with an increasing load from 0 to $500 \mathrm{mN}$; 44 measures were performed on the unmodified titanium substrate and 60 on the TiN coating. The hardness results obtained for the TiN are between 6.5 and $8.5 \mathrm{GPa}$, more than two times higher compared to that of the unmodified titanium samples, which are between 2.8 and $4.8 \mathrm{GPa}$; according these results for titanium substrates with Caram et al. ${ }^{[24]}$ Moreover, the track depths obtained for the unmodified titanium and the TiN coatings show a value of 2.7 and $1.5 \mu \mathrm{m}$, respectively, which are in agreement with the hardness results.

Micro Vickers hardness $(0.1 \mathrm{HV})$ values of the modified samples and polished titanium, as control for comparison purpose, are shown in Table 2. As seen, three hardness values for each sample are presented: surface, $(\alpha-\beta)$ diffusion area, and $(\alpha)$ unmodified structure. Values for the molybdenized surfaces, GreenTi-Mo, and SintTi-Mo, are in a range between 370 and 430, respectively. This represents hardness values one time higher than that of control titanium sample, 279.

Hardness corresponding to the $(\alpha-\beta)$ diffusion area shows a value of 281,429 , and 420 for GreenTi-Mo, SintTi-Mo, and Ti-Mo-TiN samples, respectively. This is a similar level of hardness as the value measured on the surface but higher than the values corresponding to $\alpha$ unmodified structure. Other researchers also report hardness values for CP Ti around $200 \mathrm{HV}^{[13,25]}$ Microhardness variation obtained in the different areas of the Ti-Mo samples is in a good agreement with a previous published work, where hardness is related to the Mo content. ${ }^{[13]}$ In that paper, the area with an approximately $7 \%$ of Mo content shows the highest hardness and from that point the hardness begins to decrease until values of $250 \mathrm{HV}$. Generally, $\beta$ phase alloys present higher hardness than $\alpha$ phase, thereby the introduction of Mo, $\beta$-stabilizer element, causes a small increase in hardness probably due to a higher solid solution strengthening. ${ }^{[13,14]}$

Additionally, it is possible to correlate results from Mo content (Figure 7) with those of hardness (Table 2) due to previous works where an increase in the distance from the surface involves the decrease in Mo content and the change in the crystal structure; the microhardness distribution follows the same distribution as the Mo content in the $(\alpha-\beta){ }^{[14]}$ 


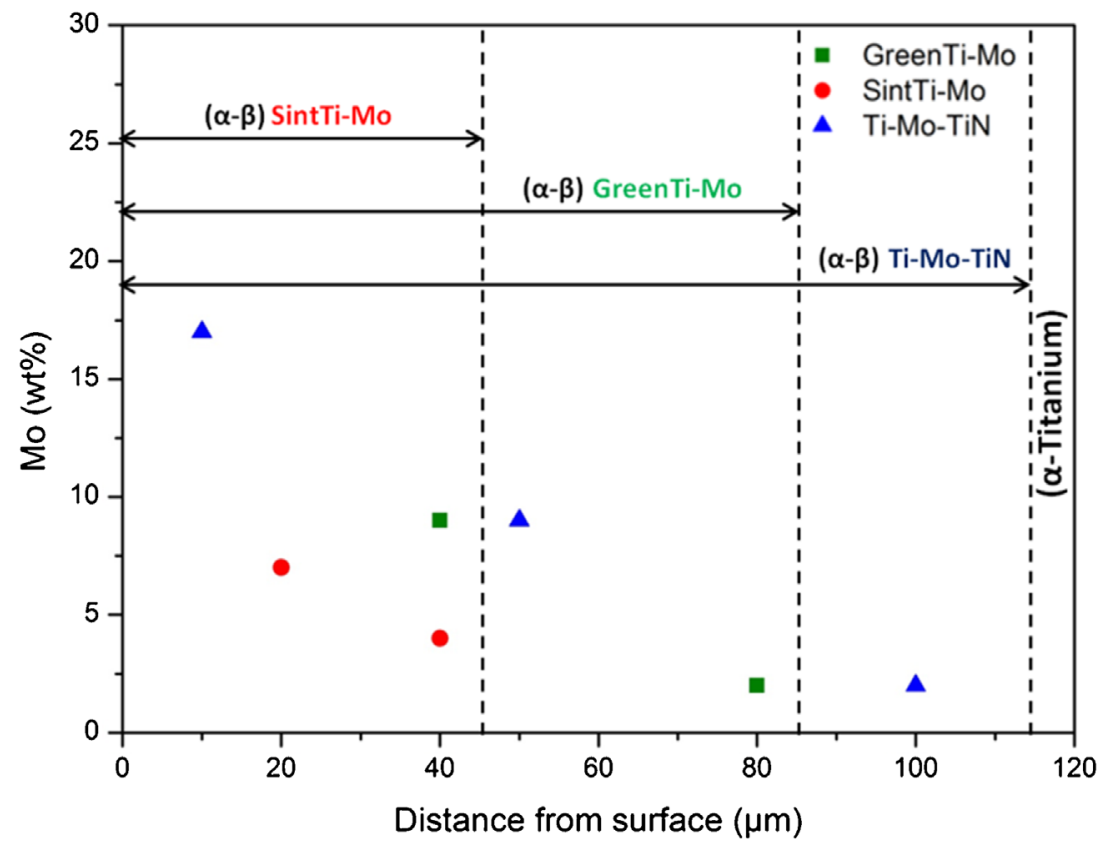

Fig. 7. Variation in Mo content of GreenTi-Mo, SintTi-Mo, and Ti-Mo-TiN samples as a function of distance from surface (energy-dispersive $\mathrm{X}$-ray spectroscopy analysis).

Considering this, hardness values for the $(\alpha-\beta)$ diffusion areas and the corresponding Mo content are as follows: $281 \mathrm{HV}, 9 \mathrm{wt} \%$ for GreenTi-Mo; $429 \mathrm{HV}, 7 \mathrm{wt} \%$ for SintTi-Mo; and $420 \mathrm{HV}, 9 \mathrm{wt} \%$ for Ti-Mo-TiN.

\section{Conclusions}

A method for producing surface modified titanium materials is presented. The surface modification is performed by $\mathrm{TiN}, \mathrm{Mo}$, and Mo-TiN coatings and the final samples are formed as a result of diffusion processes.

The TiN coating, fabricated by the low-cost and environmentally friendly technique of electrophoretic deposition, leads to homogeneous and uniform coating with a significant improvement on hardness as compared with unmodified titanium.

The Mo coating deposition is carried out onto two different titanium substrates: green compacts (only pressed) and sintered. As a result, two diffusion layers with different characteristics are obtained: i) deeper and more porous, in green compacts and ii) narrower and more homogeneous and uniform, in sintered substrates.

The surface modification by a Mo-TiN multilayer shows the thickest diffusion layer and hardest surfaces.

An initial characterization of the microstructure and hardness properties of the new created samples is also reported. Through the diffusion of molybdenum, a gradient of composition is reached. The molybdenum content decreases from the surface inward, creating a two-phase $\alpha-\beta$ region. One of the advantages of this method is found on the surface modification reached in the green compacts through the co-sintering process; saving the first sintering step of the whole process.

Based on these promising results, further investigation about the wear, corrosion, and tribocorrosion behavior of these surface modified samples is also under study to reach a better knowledge about these new designed biomaterials.

Article first published online: $x x x x$ Manuscript Revised: June 23, 2016 Manuscript Received: April 15, 2016

[1] M. Geetha, A. K. Singh, R. Asokamani, A. K. Gogia, Prog. Mater. Sci., 2009, 54, 397.

[2] S. Sedira, S. Achour, A. Avci, V. Eskizeybek, Appl. Surf. Sci. 2014, 295, 81.

[3] M. A. Hussein, A. S. Mohammed, N. Alaqeeli, S. Arabia, Materials (Basel) 2015, 8, 2749.

[4] M. T. Mohammed, Z. A. Khan, A. N. Siddiquee, Procedia Mater. Sci. 2014, 6, 1610 .

[5] A. Zhecheva, W. Sha, S. Malinov, A. Long, Surf. Coat. Technol. 2005, 200, 2192.

[6] X. Liu, P. K. Chu, C. Ding, Mater. Sci. Eng. R Rep. 2004, $47,49$.

[7] M. Niinomi, J. Mech. Behav. Biomed. Mater. 2008, 1,30 .

[8] N. Y. Kim, Y. B. Son, J. H. Oh, C. K. Hwangbo, M. C. Park, Surf. Coat. Technol. 2000, 128-129, 156.

[9] J. Morgiel, T. Wierzchoń, Surf. Coat. Technol. 2014, 259, 473.

[10] Y. Castro, B. F. C. Mendoza, Z. González, Key Eng. Mater. 2015, 654, 203.

[11] L. Besra, M. Liu, Prog. Mater. Sci. 2007, 52, 1.

[12] Y. Li, C. Yang, H. Zhao, S. Qu, X. Li, Y. Li, Materials (Basel) 2014, 7, 1709.

[13] A. Almeida, D. Gupta, C. Loable, R. Vilar, Mater. Sci. Eng. C 2012, 32, 1190.

[14] J. Li, C. Xia, Y. Gu, J. Cent. South Univ. Technol. 2004, 11, 15.

[15] X. M. Peng, C. Q. Xia, Y. Y. Liu, J. H. Wang, Surf. Coat. Technol. 2009, 203, 3306.

[16] F. F. Cardoso, P. L. Ferrandini, E. S. N. Lopes, A. Cremasco, R. Caram, J. Mech. Behav. Biomed. Mater. 2014, 32, 31.

[17] Y. H. Li, C. Yang, F. Wang, H. D. Zhao, S. G. Qu, X. Q. Li, W. W. Zhang, Y. Y. Li, Mater. Des. 2015, $85,7$.

[18] K. A. Nazari, A. Nouri, T. Hilditch, Mater. Des. 2015, 88, 1164.

[19] L. Bolzoni, P. G. Esteban, E. M. Ruiz-Navas, E. Gordo, J. Mech. Behav. Biomed. Mater. 2012, 15, 33. 
[20] L. Bolzoni, T. Weissgaerber, B. Kieback, E. M. Ruiz-Navas, E. Gordo, J. Mech. Behav. Biomed. Mater. 2013, 20, 149.

[21] R. Moreno, "Reología de suspensiones cerámicas," Madrid, 2005, 178.

[22] C. Mendoza, Z. González, Y. Castro, E. Gordo, B. Ferrari, J. Eur. Ceram. Soc. 2016, 36, 307.
[23] Y. Wang, D. Wang, J. Yan, A. Sun, Appl. Surf. Sci. 2013, $284,881$.

[24] R. Caram, J. B. Fogagnolo, A. V Rodrigues, V. Amigo, Scr. Mater. 2013, 68, 471.

[25] C. M. Lee, C. P. Ju, J. H. C. Lin, J. Oral Rehabil. 2002, 29, 314. 